\title{
Creencias de futuros y futuras docentes de inglés en Chile sobre las competencias del profesorado de inglés como lengua extranjera
}

\author{
Chilean EFL Teacher Trainees' Beliefs Regarding the EFL Teacher Competences
}

\section{Crenças de futuros docentes de inglês no Chile sobre as competências do corpo docente de inglês como língua estrangeira}
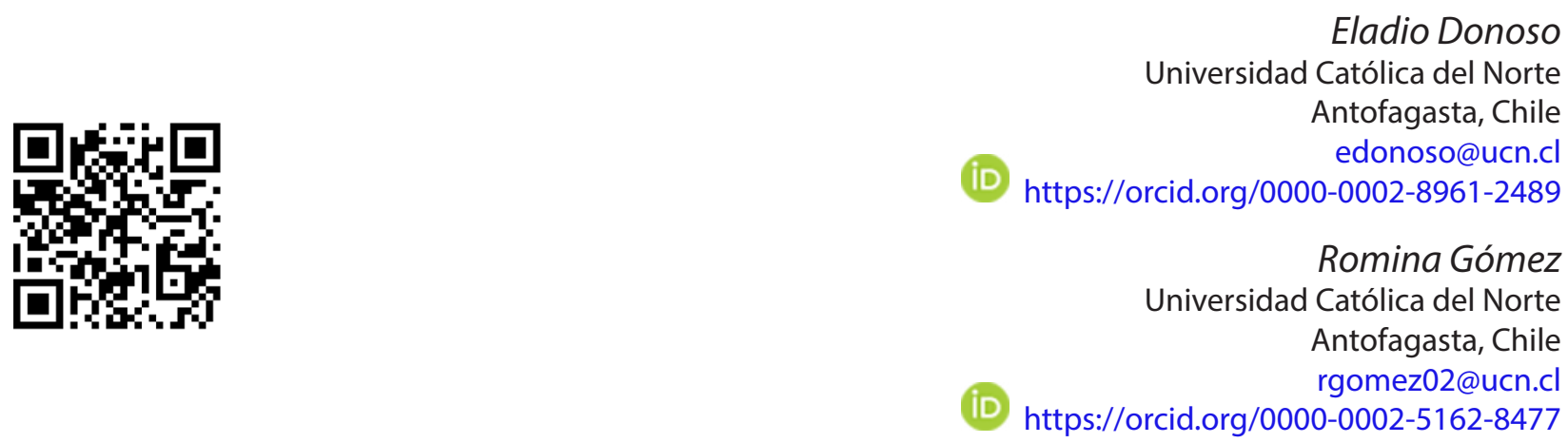

Recibido • Received • Recebido: 05 / 07 / 2019

Corregido • Revised • Revisado: 24 / 02 / 2021

Aceptado • Accepted • Aprovado: 18 / $03 / 2021$

\begin{abstract}
Resumen: Existe abundante bibliografía en la que se abordan las creencias del profesorado de segunda lengua (L2) o lengua extranjera (LE). Sin embargo, son pocos los estudios sobre este tema que consideran al profesorado de L2/LE en formación. El objetivo de este estudio es conocer las creencias que tiene el futuro profesorado de inglés respecto a las competencias que debieran identificar al profesorado de inglés como lengua extranjera (ILE) en Chile. Tres dominios de competencia están en escrutinio: (1) competencia lingüística y cultura inglesas, (2) didáctica de la enseñanza del inglés y (3) actitud profesional. Se utilizó un cuestionario tipo Likert para recopilar datos de futuro personal docente de ILE $(\mathrm{N}=208)$ que cursa primer $(\mathrm{N}=129)$ y cuarto año $(\mathrm{N}=79)$ de cuatro universidades chilenas. Los resultados generales muestran que el domino de competencia que el futuro profesorado considera más importante es el de la actitud profesional, especialmente las personas participantes de cuarto año.
\end{abstract}

Palabras claves: Creencias de docentes; formación inicial; pedagogía en inglés; inglés como lengua extranjera.

Abstract: There is abundant literature regarding second language (L2) or foreign language (FL) teachers' beliefs. However, there exists very little literature on this matter that involves $L 2 / F L$ teacher trainees. This study aims to find out Chilean EFL teacher trainees' beliefs regarding the competences EFL teachers should have for their professional performance. Three competence domains are under scrutiny: (1) English linguistic competence and English culture, (2) English teaching didactics, and 
http://doi.org/10.15359/ree.25-2.11

http://www.una.ac.cr/educare

educare@una.ac.cr

(3) professional attitude. A Likert scale questionnaire was used to collect data from $208 \mathrm{EFL}$ teacher trainees of four Chilean universities. These trainees are in their first $(\mathrm{N}=129)$ and fourth year $(\mathrm{N}=79)$ of an EFL teacher training program. General results show that the most important competence domain for the participants is the professional stance, especially for fourth year students.

Keywords: Teachers' beliefs; EFL teacher training; EFL teacher training programs in Chile; English as a foreign language.

Resumo: Existe abundante literatura na qual as crenças dos professores de segunda língua (L2) ou de língua estrangeira (LE) são abordadas. No entanto, existem poucos estudos sobre esse assunto que consideram os professores de L2 / LE que estão em formação. O objetivo deste estudo é conhecer as crenças que os futuros professores de inglês têm em relação às competências que devem identificar professores de inglês como língua estrangeira (ILE) no Chile. Três domínios de competência estão sob escrutínio: (1) proficiência e cultura da língua inglesa, (2) didática do ensino de inglês e (3) atitude profissional. Um questionário Likert foi usado para coletar dados de futuros professores da ILE (N $=208)$ que estudam primeiro $(N=129)$ e quarto ano $(N=79)$ de quatro universidades chilenas. Os resultados gerais mostram que o domínio de competência que os futuros professores consideram mais importante é a atitude profissional, especialmente entre as pessoas participantes do quarto ano.

Palavras-chave: Crenças das pessoas docentes; formação inicial de professores de ILE; pedagogia em inglês; inglês como língua estrangeira.

\section{Introducción}

En las últimas décadas se ha reconocido ampliamente que las creencias del profesorado sobre su rol profesional es uno de los factores más influyentes en su desempeño (Borg, 2012; Díaz Larenas, Alarcón Hernández y Ortiz Navarrete, 2014; Gabillon 2012b; Gilakjani, 2012; Gilakjani y Sabouri, 2017; Solís, 2015; Tatto y Coupland, 2003; Xu, 2012). Así como para otras áreas en pedagogía, existe abundante bibliografía sobre las creencias de profesores de segundas lenguas (L2) o lenguas extranjeras (LE) (Gabillon, 2012b), las cuales se han estudiado desde distintas perspectivas (Borg, 2003, 2012). Sin embargo, son pocos los estudios que examinan las creencias de docentes de L2 en formación inicial (FID), lo cual, según Levi Altstaedter y Falasca (2015), se debe a que es difícil definir el constructo. En este sentido, Gabillon (2012b) ya sostenía que esta dificultad está dada porque involucra una multitud de agentes complejos y que interactúan entre sí.

En el contexto chileno se destaca, en primer lugar, que son insuficientes los estudios que abordan la FID del profesorado de inglés (Barahona, 2016), lengua extranjera que se enseña predominantemente en el país. Cabe subrayar, sin embargo, que los escasos hallazgos sobre creencias del estudiantado de pedagogía en inglés en Chile acentúan que el origen de estas mismas está dado por las experiencias que tuvieron como estudiantes de lengua extranjera y, de igual forma, se observa que dichas creencias van variando a partir de su aprendizaje en la práctica pedagógica (Barahona, 2016; Díaz Larenas, Alarcón Hernández, Vergara et al., 2014).

2 
Ahora bien, el profesorado de una L2, como coprotagonistas del proceso de enseñanzaaprendizaje de esta, debe demostrar una sólida preparación disciplinar, pedagógica y didáctica (Martínez Agudo, 2011). Así las cosas, resulta de interés el indagar en las creencias de estudiantes de pedagogía en inglés acerca de competencias lingüísticas, didácticas y actitudinales que estos grupos consideran debiera tener un docente de inglés como lengua extranjera (ILE) ya con formación completada y en ejercicio, lo anterior teniendo en cuenta al profesorado como mediador del acto didáctico y su importancia en el proceso formativo de sus estudiantes.

A partir de lo anterior, el presente artículo informa los resultados de un estudio que se inscribe dentro de un enfoque metodológico cuantitativo, descriptivo y transeccional, cuyo objetivo general es determinar las creencias de estudiantes en FID de ILE de cuatro universidades chilenas, de primer y cuarto año, acerca de las competencias que debería tener un profesor o profesora de ILE en tres dominios: 1) competencia lingüística y cultura inglesas, (2) didáctica de la enseñanza del inglés y (3) actitud profesional. De igual forma, se espera observar si existen diferencias en dichas creencias, dependiendo del año que cursen las personas participantes en su plan de estudios y la universidad en la que lo cursan. Lo precedente se efectúo a través de la aplicación de un cuestionario tipo Likert y en el marco de la línea de investigación conocida como Teacher's beliefs.

\section{Referente teórico}

Como ya se ha señalado, creencia es un constructo difícil de definir (Gabillon, 2012b; Levi Altstaedter y Falasca, 2015). Pajares (1992), basándose en investigaciones anteriores sobre el tema, sostiene que creencia es un messy concept. Es por ello que Pajares (1992) sostiene que ellas viajan disfrazadas y a menudo bajo alias: actitudes, valores, juicios, axiomas, opiniones, ideología, percepciones, concepciones, sistemas conceptuales, preconcepciones, disposiciones, teorías implícitas, teorías explícitas, teorías personales, procesos mentales internos, estrategias de acción, reglas de la práctica, principios prácticos, perspectivas, repertorios de entendimiento y estrategia social, por nombrar solo algunas que se pueden encontrar en la bibliografía.

A estos veintiún términos expuestos por Pajares (1992), la bibliografía especializada suma muchas y variadas definiciones de creencia (Ver Gilakjani y Sabouri, 2017). En esta línea, Ramos Méndez (2007, pp. 17-18) perfila algunos rasgos de las creencias, a saber:

Son ideas muy estables que forman parte integral y orgánica de nuestro pensamiento (por tanto, estamos convencidos de que son verdad); además, tienen valor interpretativo y evaluativo, puesto que nos ayudan a enfrentarnos al mundo, guiando y orientando nuestra acciones y conductas, sean de carácter intelectual o no.

Ahora bien, en el ámbito de la educación, se sostiene que las creencias pedagógicas corresponden a redes complejas de procesamiento almacenadas en la memoria a largo plazo -memorias semánticas y episódicas- (Díaz Larenas y Solar Rodríguez, 2011). De igual forma, se 
http://doi.org/10.15359/ree.25-2.11

http://www.una.ac.cr/educare

educare@una.ac.cr

consideran estables, rígidas y difícilmente modificables. A este tipo de creencias se las denomina core beliefs o creencias centrales y fosilizadas. Estas son dominantes, uniformes, permanentes, resistentes, sociales, explícitas y sistemáticas. Por otro lado, están, también, las denominadas peripheral beliefs, las que se consideran como subordinadas, moldeables, diversas, variables, personales, implícitas y complejas, por lo tanto, menos estables (Gabillon, 2012b, Gilakjani, 2012; Gilakjani y Sabouri, 2017; Moscovici, 1998).

Enfocada específicamente a la enseñanza de L2, como ya se ha sostenido, existe abundante bibliografía. Esta da cuenta de que el fenómeno se ha abordado desde distintos ángulos y ha considerado distintos actores. A modo de ejemplo, Borg (2003) llevó a cabo un estudio de 64 publicaciones y concluyó que existen notorias diferencias entre docentes respecto de la enseñanza y aprendizaje de una FL o L2, esto enfocado, principalmente, en la gramática. Por su parte, Gilakjani y Sabouri (2017) reportan numerosos estudios en relación con, entre otros aspectos, la conexión entre la práctica de aula y las creencias del profesorado en servicio, las creencias del personal docente y sus planificaciones, las creencias de profesorado en formación y profesorado en servicio respecto a sus estrategias de enseñanza, las creencias referente a la importancia de la gramática en L2, diferencias entre las creencias de profesorado novel y experimentado y la influencia de programas de formación en el cambio en las creencias. Es así que los autores concluyen que las creencias del profesorado están relacionadas con varios factores que interactúan entre sí y que se forman o adquieren durante los muchos años de experiencias de aprendizajes, teniendo un efecto en cómo aprenden a enseñar. Sostienen, además, que las creencias tienen un impacto en el quehacer del profesorado ya que están involucradas sus actitudes, toma de decisiones, planificación y métodos de enseñanza, entre otros, lo que obviamente afecta el aprendizaje del estudiantado.

En relación con el término 'creencia', Ferreira Barcelos (2007) argumenta que este refiere a una serie de conceptos provenientes de distintas corrientes de investigación. Así, Ferreira Barcelos (2007) realiza una lista de diferentes términos utilizados por distintos autores, sosteniendo que definir creencias sobre la adquisición de segunda lengua es difícil porque, al igual que con las creencias en general, se han usado varios términos para referirse a ellas. En este mismo ámbito y de acuerdo con Gabillon (2012b), las creencias teórico profesionales del profesorado de L2 se construyen, primero, en la escuela, como estudiantes-aprendientes de una lengua; pero, más tarde, estas se convierten en la base para las que se construyen en el contexto formativo y profesional. A este respecto, Gabillon (2012b) añade que las creencias teórico-pedagógicas del profesorado de segunda lengua se construyen mucho más tarde en la vida. Esto ocurre durante su formación profesional a través de su interacción con otros y otras estudiantes de pedagogía y con su personal formador de profesionales. Las creencias que se forman a más temprana edad son los prototipos que sirven como referencia cuando estos profesorados comienzan a construir sus creencias respecto a su profesión.

La interrogante que surge a partir de la afirmación de Gabillon (2012b) es qué ocurre en el contexto chileno; qué creencias 'construyen' los futuros profesorados de inglés durante su formación profesional; se advierten diferencias significativas dependiendo de la institución 
http://doi.org/10.15359/ree.25-2.11

formadora. El estudio que se presenta en este artículo da cuenta de las respuestas a estas interrogantes. Para ello se emplea el método que se describe a continuación.

\section{Metodología}

La metodología está dada en razón de los siguientes objetivos planteados:

\section{Objetivo general}

Determinar las creencias de estudiantes de primer y cuarto año de la carrera de pedagogía en inglés de cuatro universidades chilenas respecto de los dominios de competencia, que ellos creen, deben definir a un profesor o profesora de inglés que se forma en Chile.

\section{Objetivos específicos}

1. Establecer si existen diferencias entre las creencias del estudiantado de primer año de formación inicial docente respecto de sus pares de cuarto año.

2. Determinar si existen diferencias entre las creencias del estudiantado dependiendo de la universidad en la que cursan sus estudios.

\section{Diseño de investigación}

Para llevar a cabo la investigación que se reporta en este artículo, se realizó un diseño no experimental, descriptivo y transeccional, puesto que se abordó a las personas participantes en un punto específico en el tiempo (Hernández Sampieri et al., 2014).

\section{Muestra de población y contexto del estudio}

La muestra es de tipo no probabilístico, por conveniencia (Hernández Sampieri et al., 2014). Está compuesta por 208 estudiantes universitarios, 129 de primer año, cuyas edades fluctúan entre los 18 y 21 años, y 79 de cuarto año, con edades de entre 21 y 25 años, de la carrera de Pedagogía en Inglés de cuatro universidades chilenas acreditadas por el sistema de acreditación nacional chileno y pertenecientes al Consejo de Rectores de Universidades Chilenas (CRUCH) de cuatro regiones del país: Arica y Antofagasta ubicadas en el norte de Chile y regiones de El Maule y Ñuble del centro sur.

Se elige el estudiantado de primer y cuarto año puesto que se podrían obtener resultados más robustos para poder establecer, si existen diferencias entre aquellos sujetos que recién comienzan su formación inicial docente - primer año- con respecto a quienes llevan más tiempo de preparación y están a un par de semestres de graduarse. Con dichos resultados, se podría inferir que el proceso de formación inicial docente influye o no en el cambio de las creencias de los sujetos participantes del estudio. 
http://doi.org/10.15359/ree.25-2.11

http://www.una.ac.cr/educare

educare@una.ac.cr

Las cuatro casas de estudios superiores presentan en común los mismos ejes y dominios de competencia en sus currículos para la FID de profesores ILE (Abrahams y Farías, 2010; Ministerio de Educación, Gobierno de Chile, 2013). Estas consideran, principalmente, las competencias relacionadas con, primero, la disciplina (lingüística, literatura y cultura inglesas) (al que para este estudio hemos denominado dominio de lingüística y cultura inglesas); segundo, el desarrollo de habilidades relacionadas con la metodología y didáctica (denominado aquí dominio de didáctica de la enseñanza del inglés) y, tercero, la competencia pedagógica en sí, la cual involucra una serie de conocimientos, capacidades, habilidades, destrezas, actitudes que posee el profesorado para intervenir en la orientación integral de sus estudiantes (llamado aquí dominio actitud profesional). En consideración a lo anterior, se debe explicitar que las cuatro universidades otorgan el título profesional de Profesor o Profesora de Inglés y el grado académico de Licenciatura en Educación.

\section{Instrumento}

Los datos fueron obtenidos por medio de un cuestionario en escala Likert (ver Apéndice A), elaborado por las personas investigadoras y validado por juicio experto. Dicho cuestionario presenta 50 afirmaciones distintas (reactivos) respecto a los tres dominios: lingüística y cultura inglesa (15 afirmaciones), didáctica de la enseñanza del inglés (16 afirmaciones) y actitud profesional (19 afirmaciones). Ante cada afirmación, las personas participantes debían indicar su nivel de acuerdo con las opciones muy en desacuerdo, en desacuerdo, indiferente, de acuerdo y muy de acuerdo.

Para efectos de validez, se incluyeron preguntas abiertas y se realizó un pilotaje con la participación de estudiantes de segundo y tercer año de la carrera de pedagogía en inglés de una de las cuatro universidades consideradas para el estudio.

Para proteger los aspectos éticos de la investigación, se les solicitó a las personas informantes que firmaran un consentimiento informado. La aplicación del instrumento se dio a conveniencia del horario de los cursos del estudiantado, lo que significó utilizar aproximadamente 45 minutos de una de sus clases.

\section{Proceso de análisis y resultados}

Una vez aplicado el instrumento, se procedió a la tabulación y posterior análisis estadístico de los datos. Dichos análisis se muestran a continuación. dominio.

En primer lugar, en la Tabla 1, se presentan los estadísticos descriptivos globales de cada 
Tabla 1: Descriptivos globales para dominios estudiados

\begin{tabular}{lcccccc}
\hline & Mínimo & Máximo & Media & D.E. & Asimetría & Curtosis \\
\hline Competencia lingüística y cultura inglesas & 2.57 & 5.00 & 4.31 & .47 & -1.11 & 1.72 \\
Didáctica de la enseñanza del inglés & 3.00 & 5.00 & 4.40 & .45 & -.82 & .36 \\
Actitud profesional & 3.11 & 5.00 & 4.51 & .38 & -1.14 & 1.33 \\
\hline
\end{tabular}

$\mathrm{n}=208$

Nota: Elaboración propia.

Se puede apreciar que el dominio actitud profesional presenta los resultados más altos y, al mismo tiempo, es el que posee la menor dispersión (D.E.=.38). Esto se puede interpretar como una tendencia sistemática a evaluar de mejor manera los ítems de este dominio. Sin embargo, es importante recalcar que todos los dominios se encuentran en un nivel más bien alto.

Del mismo modo, se procedió a comparar los dominios por nivel de curso, esto es, entre quienes están en primer y en cuarto año, lo que se observa en las Tablas 2 y 3.

Tabla 2: Descriptivos para dominios estudiados por año en curso y prueba de diferencia de medias

\begin{tabular}{llllllll}
\hline & & $\mathrm{N}$ & Media & Desviación típ. & $\mathrm{T}$ & $\mathrm{p}$ \\
\hline \multirow{2}{*}{ Lingüística y cultura inglesas } & Primer año & 129 & 4.37 & .49 & \multirow{2}{*}{2.34} & \multirow{2}{*}{0.02} \\
\cline { 2 - 6 } & Cuarto año & 79 & 4.22 & .41 & & \multirow{2}{*}{2.56} & \multirow{2}{*}{0.01} \\
\hline \multirow{2}{*}{ Didáctica de la enseñanza del inglés } & Primer año & 129 & 4.46 & .49 & & \multirow{2}{*}{-2.04} & \multirow{2}{*}{0.04} \\
\cline { 2 - 6 } Actitud profesional & Cuarto año & 79 & 4.31 & .36 & & \\
\hline \multirow{2}{*}{ Crimer año } & 129 & 4.47 & .39 & & & \\
\cline { 2 - 6 } & Cuarto año & 79 & 4.58 & .35 & & & \\
\hline
\end{tabular}

Nota: Elaboración propia.

Tabla 3: Descriptivos de forma para dimensiones estudiadas por año en curso

\begin{tabular}{llcc}
\hline \multirow{2}{*}{ Lingüística y cultura inglesas } & Asimetría & Curtosis \\
\hline \multirow{2}{*}{ Didáctica de la enseñanza del inglés } & -1.35 & 2.09 \\
\cline { 2 - 4 } & Cuarto año & -.89 & 2.04 \\
\cline { 2 - 4 } & Primer año & -1.08 & .58 \\
\hline \multirow{2}{*}{ Actitud profesional } & Cuarto año & -.49 & .49 \\
\cline { 2 - 4 } & Primer año & -.94 & .75 \\
\hline
\end{tabular}

Nota: Elaboración propia. 
http://doi.org/10.15359/ree.25-2.11

http://www.una.ac.cr/educare

educare@una.ac.cr

Se pudo encontrar diferencias significativas en todas las dimensiones $(p<.05)$. En el caso del dominio lingüística y cultura inglesas y el dominio didáctica de la enseñanza del inglés, fueron los primeros años quienes lo evaluaron con mayor importancia, mientras que el dominio actitud profesional fue el estudiantado de cuarto año el que le asignó la media más alta.

Respecto de las medidas de forma, se puede apreciar que existe una tendencia a que las respuestas sean más positivas en los cursos de primer año, excepto en el tercer dominio, donde la tendencia es más marcada en cuarto año.

Al analizar las curtosis, destacan el primer dominio, donde tanto en primero como en cuarto año, la tendencia es a tener una curtosis alta. Esto implica que los datos se concentran fuertemente en torno a la media. En el tercer dominio, llama la atención que en cuarto año la curtosis es especialmente alta respecto de primer año. Esto denota que hay una marcada y clara concentración de datos en torno a una media alta, lo cual no es tan notable en primer año. Este dominio es evaluado de manera mucho más positiva en cuarto año. Esto es importante de notarlo con el fin de conocer más a fondo las diferencias halladas en los análisis de la Tabla 2.

En cuanto a las dimensiones según universidad, no se encontraron diferencias estadísticamente significativas entre ellas, tal como lo muestran las Tablas 4 y 5 . Esto quiere decir que todos los sujetos de las cuatro casas de estudio que constituyen la muestra valoran de la misma manera estas variables.

Tabla 4: Descriptivos para dominios estudiados por universidad y prueba de diferencia de medias

\begin{tabular}{|c|c|c|c|c|c|c|}
\hline Universidad & & $\mathrm{n}$ & Media & D.E & $\mathrm{F}$ & $\mathrm{p}$ \\
\hline \multirow{4}{*}{ Lingüística y cultura inglesas } & U-1 & 63 & 4.29 & .47 & \multirow{4}{*}{1.288} & \multirow{4}{*}{0.28} \\
\hline & U-2 & 54 & 4.42 & .35 & & \\
\hline & $U-3$ & 64 & 4.27 & .52 & & \\
\hline & $\mathrm{U}-4$ & 27 & 4.25 & .50 & & \\
\hline \multirow{4}{*}{ Didáctica de la enseñanza del inglés } & $U-1$ & 63 & 4.50 & .43 & \multirow{4}{*}{2.136} & \multirow{4}{*}{0.09} \\
\hline & $\mathrm{U}-2$ & 54 & 4.42 & .36 & & \\
\hline & $U-3$ & 64 & 4.33 & .51 & & \\
\hline & $U-4$ & 27 & 4.30 & .46 & & \\
\hline \multirow{4}{*}{ Actitud profesional } & $\mathrm{U}-1$ & 63 & 4.56 & .41 & \multirow{4}{*}{2.669} & \multirow{4}{*}{0.05} \\
\hline & U-2 & 54 & 4.58 & .23 & & \\
\hline & U-3 & 64 & 4.48 & .41 & & \\
\hline & U-4 & 27 & 4.36 & .42 & & \\
\hline
\end{tabular}

Nota: Elaboración propia. 
http://doi.org/10.15359/ree.25-2.11

Tabla 5: Descriptivos de forma para dimensiones estudiadas por universidad

\begin{tabular}{lccc}
\hline \multirow{2}{*}{ Universidad } & & Asimetría & Curtosis \\
\hline \multirow{3}{*}{ Lingüística y cultura inglesas } & $\mathrm{U}-1$ & -.95 & 1.15 \\
\cline { 2 - 4 } & $\mathrm{U}-2$ & -.31 & -.84 \\
\cline { 2 - 4 } & $\mathrm{U}-3$ & -1.09 & 1.22 \\
\cline { 2 - 4 } & $\mathrm{U}-4$ & -1.57 & 3.61 \\
\cline { 2 - 4 } Didáctica de la enseñanza del inglés & $\mathrm{U}-1$ & -1.08 & .99 \\
\cline { 2 - 4 } & $\mathrm{U}-2$ & -.20 & -.84 \\
\cline { 2 - 4 } & $\mathrm{U}-3$ & -.77 & -.16 \\
\cline { 2 - 4 } & $\mathrm{U}-4$ & -.70 & -.01 \\
\cline { 2 - 4 } Actitud profesional & $\mathrm{U}-1$ & -1.07 & -.23 \\
\cline { 2 - 4 } & $\mathrm{U}-2$ & -.53 & 1.61 \\
\cline { 2 - 4 } & $\mathrm{U}-3$ & -1.17 & 1.51 \\
\cline { 2 - 4 } & $\mathrm{U}-4$ & -.89 & \\
\hline
\end{tabular}

Nota: Elaboración propia.

Respecto de las medidas de forma, se puede apreciar que en general las asimetrías son en algunos casos altas, pero no superiores a 2. Esto implica que, si bien se aprecia una tendencia a que las respuestas sean positivas, esta tiende a ser más marcada solo en ciertos casos. No se aprecia una tendencia a una universidad específica. Una situación similar ocurre con la curtosis, donde destaca el caso del dominio actitud profesional en la U-3 y U-4, donde los datos se concentran en mayor medida en torno a la media.

\section{Discusión y conclusión}

El propósito del presente estudio fue determinar las creencias, y posibles diferencias entre estas, de estudiantes en formación inicial de pedagogía en inglés de cuatro universidades a lo largo de Chile de primer y cuarto año, acerca de los dominios de competencias que debería tener un profesor o profesora de ILE en la lengua inglesa, la didáctica y la actitud profesional en su desempeño docente. De esta manera se pretende determinar: si estas creencias se construyen (Gabillon, 2012b), son periféricas (Gabillon, 2012a; Moscovici, 1998) y cambian durante la formación profesional. De igual manera, se esperaba detectar diferencias entre universidades respecto a su distribución geográfica a lo largo del país. Para lo anterior se utilizó una muestra por conveniencia de 208 estudiantes de cuatro universidades chilenas de primer y cuarto años de sus programas de estudios y se les aplicó un cuestionario escala Likert para que expresaran su nivel de acuerdo o desacuerdo a 50 reactivos.

Los resultados indican, en términos generales, es decir, sin considerar la universidad o año que cursan las personas participantes, que el dominio actitud profesional es el que más destaca. 
http://doi.org/10.15359/ree.25-2.11

http://www.una.ac.cr/educare

educare@una.ac.cr

Ahora bien, considerando solo el año que cursan los sujetos, se observa que los de primer año otorgan mayor importancia y con diferencias estadísticamente significativas a los dominios lingüística y cultura inglesas y didáctica de la enseñanza del inglés por sobre el de actitud profesional. Esto implica que los sujetos de cuarto año confieren mayor valor al dominio del profesorado en cuanto a su desenvolvimiento personal y profesional y no a lo que enseña o cómo lo enseña (Ver Tabla 3). De igual forma, es posible pensar, a la luz de lo que afirma Gabillon (2012b), que el alumnado de primer año de formación como profesor o profesora ILE se adhieren con mayor fuerza a las creencias que se formaron como estudiantes-aprendientes de la lengua en la escuela y que estas cambian producto de la formación universitaria. Se podría, también, y a pesar que este es un estudio transeccional, inferir que las creencias del futuro profesorado que constituye la muestra varía a medida que avanzan en su formación como docente, es decir, son más bien peripherical beliefs (Gabillon, 2012b; Moscovici, 1998), coincide, asimismo, con lo planteado por Barahona (2016) y Díaz Larenas, Alarcón-Hernández, Vergara et al. (2014), en tanto y en cuanto las creencias del futuro profesorado ILE son dinámicas, es decir, varían en el tiempo.

En cuanto a las dimensiones según universidad, los resultados no muestran diferencias significativas. Este hallazgo resulta interesante, puesto que esto sucede no obstante que las casas de estudios están ubicadas en zonas de Chile que presentan importantes diferencias étnicas, sociales y culturales entre sí; destaca, por ejemplo, que U-4 que se ubica en el extremo norte del país distante $2440 \mathrm{kms}$ de la U-1. Con esto se podría sostener que existe homogeneidad en la formación inicial de docentes de ILE, lo que se podría atribuir a que las instituciones formadoras se adhieren a la política pública nacional para la FID de profesorado ILE, lo que lo permea para su futuro profesional.

Desde el quehacer como personal investigador y formador de docentes ILE, creemos que este estudio puede brindar información que contribuya a la reflexión entre docentes, entre estudiantes, y entre docentes y estudiantes. De igual forma, puede contribuir a proponer y establecer prioridades de pertinencia y mejora de, por ejemplo, el currículo, contenidos y resultados de aprendizajes; todo ello tendiendo a la consolidación de competencias que contribuyan a fortalecer el desempeño del futuro profesor y profesora.

\section{Declaración de procedencia}

Este artículo deriva del Proyecto Semilla UCN2017, Vicerrectoría de Investigación y Desarrollo Tecnológico, Universidad Católica del Norte.

\section{Declaración de Material complementario}

Este artículo tiene disponible, como material complementario:

-La versión preprint del artículo en https://doi.org/10.5281/zenodo.3922900 
http://doi.org/10.15359/ree.25-2.11

\section{Referencias}

Abrahams, M. J. y Farias, M. (2010). Struggling for change in chilean EFL teacher education. ColombianApplied Linguistics Journal, 12(2), 110-118.https://doi.org/10.14483/22487085.87

Barahona, M. (2014). Pre-service teachers' beliefs in the activity of learning to teach English in the chilean context. Cultural-Historical Psychology, 10(2), 116-12. https://psyjournals.ru/ files/70042/kip 2014 2 barahona.pdf

Barahona, M. (2016). English language teacher education in Chile. A cultural historical activity theory perspective. Routledge. https://doi.org/10.4324/9781315689937

Borg, S. (2003). Teacher cognition in language teaching: A review of research on what language teachers think, know, believe and do. Language Teaching, 36(2), 81-109. https://doi. org/10.1017/S0261444803001903

Borg, S. (2012). Current approaches to language teacher cognition research: A methodological analysis. En R. Barnard y A. Burns (Eds.), Researching language teacher cognition and practice. International case studies (pp. 11-29). Multilingual Matters. https://doi. org/10.21832/9781847697912-003

Díaz Larenas, C., Alarcón Hernández, P., Vergara, J., Ortiz, M. y Tagle, T. (2014). Creencias de estudiantes de pedagogía en inglés sobre los modelos didácticos utilizados para la enseñanza del idioma. Núcleo, 26(31), 41-74. http://ve.scielo.org/scielo.php?script=sci_art text\&pid=S0798-97842014000100002

Díaz Larenas, C., Alarcón Hernández, P. y Ortiz Navarrete, M. (2014). A case study on EFL teachers' beliefs about the teaching and learning of English in public education. Porta Linguarum, 23, 171-186. http://hdl.handle.net/10481/53765

DíazLarenas,C.ySolarRodríguez,M.I.(2011).Larevelacióndelascreenciaslingüístico-pedagógicas a partir del discurso del profesor de inglés universitario. RIA. Revista de Lingüística Teórica y Aplicada, 49(2), 57-86. https://doi.org/10.4067/S0718-48832011000200004

Ferreira Barcelos, A. M. (2007). Researching beliefs about SLA: A critical review. En P. Kalaja y A. M. Ferreira Barcelos (Eds.), Beliefs about SLA: New research approaches (pp. 7-33). Kluwer Academic Publishers. https://doi.org/10.1007/978-1-4020-4751-0_1

Gabillon, Z. (2012a). Discrepancies between L2 Teacher and L2 Learner Beliefs. English Language Teaching, 5(12), 94-99. https://doi.org/10.5539/elt.v5n12p94

Gabillon, Z. (2012b). Revisiting foreign language teacher beliefs. Frontiers of Language and Teaching, 3, 190-203. https://www.researchgate.net/publication/259803382 Revisiting Foreign Language Teacher Beliefs 
http://doi.org/10.15359/ree.25-2.11

http://www.una.ac.cr/educare

educare@una.ac.cr

Gilakjani, A. P. (2012). EFL teachers' beliefs toward using computer technology in English language teaching. Journal of Studies in Education, 2(2), 62-80. https://doi.org/10.5296/jse. v2i2.1174

Gilakjani, A. P. y Sabouri, N. B. (2017). Teachers' beliefs in English language teaching and learning: A review of the literature. English Language Teaching, 10(4), 78-86. https://doi.org/10.5539/ elt.v10n4p78

Hernández Sampieri, R., Fernández Collado, C. y Baptista Lucio, P. (2014). Metodología de la investigación (6. ed.). McGraw-Hill.

Levi Altstaedter, L y Falasca, M. (2015). Exploring foreign language pre-service teacher beliefs: An international exchange experience. Global Partners in Education Journal, 5(1), 38-50. http://www.gpejournal.org/index.php/GPEJ/article/view/91

Martínez Agudo, J. de D. (2011). Perfil profesional idóneo del profesor de lengua extranjera: Creencias del profesorado en formación. Revista Latinoamericana de Estudios Educativos, 41(1-2) 103-124. http://www.redalyc.org/articulo.oa?id=27021144004

Ministerio de Educación, Gobierno de Chile. (2013). Estándares orientadores para carreras de pedagogíaeninglés.Autor.https://bibliotecadigital.mineduc.cl/handle/20.500.12365/2237

Moscovici, S. (1998). The history and actuality of social representations. En S. Moscovici y G. Duveen (Eds.), Social representations: Explorations in social psychology (pp. 120-155). Polity Press.

Pajares,M.F.(1992).Teachers'beliefsandeducationalresearch:Cleaningupamessyconstruct.Review of Educational Research, 62(3), 307-332. https://doi.org/10.3102/00346543062003307

Ramos Méndez, C. (2007). El pensamiento de los aprendientes en torno a cómo se aprende una lengua: Dimensiones individuales y culturales. Secretaría General Técnica.

Solís, C. A. (2015). Creencias sobre enseñanza y aprendizaje en docentes universitarios: Revisión de algunos estudios. Propósitosy Representaciones, 3(2), 227-260. https://doi.org/10.20511/ pyr2015.v3n2.83

Tatto, M. T. y Coupland, D. B. (2003). Teacher education and teachers' beliefs. Theoretical and measurement concerns. En J. Raths y A. C. McAninch (Eds.), Teacher beliefs and classroom performance: The impact of teacher education (pp. 123-182). Information Age Publishing.

$\mathrm{Xu}, \mathrm{L}$. (2012). The role of teachers' beliefs in the language teaching-learning process. Theory and Practice in Language Studies, 2(7), 1397-1402. https://doi.org/10.4304/tpls.2.7.1397-1402 
http://doi.org/10.15359/ree.25-2.11

Apéndice A: Creencias de estudiantes en formación inicial de pedagogía en inglés acerca de competencias del profesorado de inglés como lengua extranjera

\section{SECCIÓN I. CREENCIAS}

Marca con una $\mathbf{X}$ la alternativa que expresa mejor tu opinión respecto de cada información, considerando que 1 es "Muy en desacuerdo" y 5 es "Muy de acuerdo". En caso que no esté clara la afirmación, puedes dejarla en blanco.

1. Identifica ideas principales de los textos que lee en inglés.

2. Tiene un buen manejo de la gramática inglesa.

3. Posee amplio conocimiento de la cultura de países donde se habla inglés.

4. Realiza evaluaciones que involucren las cuatro habilidades del inglés.

5. Hace que el alumnado lea textos en inglés y revisa si comprenden lo que leyeron.

6. Practica la pronunciación en la clase a través de repetición grupal o individual.

7. Se da cuenta de sus propios errores y los corrige.

8. Considera los intereses de sus estudiantes en la planificación de la clase.

9. Respeta las opiniones de todo el estudiantado.

10. Da fórmulas gramaticales para construcción de oraciones en inglés.

11. Conoce refranes, modismos y dichos típicos de la lengua inglesa.

12. Promueve el desarrollo del pensamiento.

13. Tiene muy buena pronunciación en inglés.

14. Planifica la clase de manera que se ejerciten las cuatro habilidades del inglés.

15. Establece un ambiente organizado de trabajo en la clase.

16. Motiva a sus estudiantes al logro de los aprendizajes.

17. Reflexiona constantemente sobre cómo hace sus clases.

18. Presenta vocabulario nuevo en contexto que facilite su aprendizaje.

19. Maneja distintos tipos de inglés (por ejemplo, británico, estadounidense)

20. Propone actividades interesantes y desafiantes para que el estudiantado aprenda.

21. Manifiesta a sus estudiantes lo que espera de ellos y ellas.
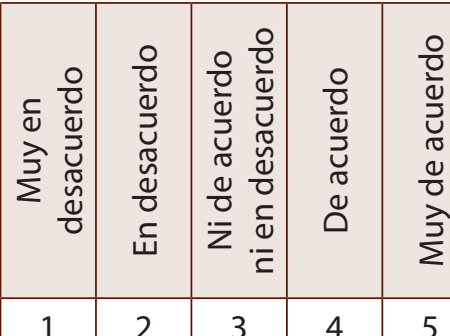

\begin{tabular}{|l|l|l|l|l|}
1 & 2 & 3 & 4 & 5 \\
\hline 1 & 2 & 3 & 4 & 5 \\
\hline
\end{tabular}


http://doi.org/10.15359/ree.25-2.11

http://www.una.ac.cr/educare

educare@una.ac.cr

\begin{tabular}{|c|c|c|c|c|c|}
\hline $\begin{array}{l}\text { 22. Utiliza material auténtico (revistas, diarios, videos, etc.) para las actividades } \\
\text { de la clase. }\end{array}$ & 1 & 2 & 3 & 4 & 5 \\
\hline 23. Evalúa a sus estudiantes de forma ecuánime, justa y objetiva. & 1 & 2 & 3 & 4 & 5 \\
\hline 24. Produce correctamente discurso escrito en inglés. & 1 & 2 & 3 & 4 & 5 \\
\hline 25. Comprende apropiadamente discurso oral y escrito en inglés. & 1 & 2 & 3 & 4 & 5 \\
\hline 26. Tiene amplio dominio de vocabulario. & 1 & 2 & 3 & 4 & 5 \\
\hline 27. Domina aspectos pragmáticos de la lengua inglesa (ironía, humor). & 1 & 2 & 3 & 4 & 5 \\
\hline $\begin{array}{l}\text { 28. Realiza actividades que permiten a todo el estudiantado hablar en inglés } \\
\text { durante la clase. }\end{array}$ & 1 & 2 & 3 & 4 & 5 \\
\hline 29. Considera los diversos ritmos de aprendizaje de sus estudiantes. & 1 & 2 & 3 & 4 & 5 \\
\hline 30. Tiene un trato amable con sus estudiantes. & 1 & 2 & 3 & 4 & 5 \\
\hline 31. Conoce a sus estudiantes y les llama por su nombre. & 1 & 2 & 3 & 4 & 5 \\
\hline 32. Promueve un ambiente distendido en la clase. & 1 & 2 & 3 & 4 & 5 \\
\hline 33. Organiza actividades de aprendizaje por equipos. & 1 & 2 & 3 & 4 & 5 \\
\hline 34. Optimiza el tiempo disponible para la enseñanza. & 1 & 2 & 3 & 4 & 5 \\
\hline 35. Inspira confianza para que sus estudiantes comuniquen sus inquietudes. & 1 & 2 & 3 & 4 & 5 \\
\hline 36. Despliega una diversidad de actividades que promueven el uso del inglés. & 1 & 2 & 3 & 4 & 5 \\
\hline 37. Sabe literatura inglesa clásica. & 1 & 2 & 3 & 4 & 5 \\
\hline 38. Domina con propiedad la ortografía del inglés. & 1 & 2 & 3 & 4 & 5 \\
\hline 39. Explica muy bien la gramática. & 1 & 2 & 3 & 4 & 5 \\
\hline 40. Entrega listas de vocabulario para aprender. & 1 & 2 & 3 & 4 & 5 \\
\hline 41. Hace que el estudiantado escriba en inglés cartas, ensayos, resúmenes, etc. & 1 & 2 & 3 & 4 & 5 \\
\hline $\begin{array}{l}\text { 42. Organiza actividades de conversación y uso oral del inglés (diálogos, } \\
\text { disertaciones, etc.). }\end{array}$ & 1 & 2 & 3 & 4 & 5 \\
\hline $\begin{array}{l}\text { 43. Realiza actividades de comprensión auditiva como, por ejemplo, que el } \\
\text { estudiantado dibuje lo que oye. }\end{array}$ & 1 & 2 & 3 & 4 & 5 \\
\hline 44. Establece autoridad a partir de su formalidad al vestir. & 1 & 2 & 3 & 4 & 5 \\
\hline 45. Genera oportunidades de aprendizaje para sus estudiantes. & 1 & 2 & 3 & 4 & 5 \\
\hline $\begin{array}{l}\text { 46. Usa películas, documentales o material audiovisual en inglés para que } \\
\text { luego sus estudiantes los comenten y analicen. }\end{array}$ & 1 & 2 & 3 & 4 & 5 \\
\hline $\begin{array}{l}\text { 47. Provee listado de verbos irregulares para que los estudiantes y las } \\
\text { estudiantes los aprendan. }\end{array}$ & 1 & 2 & 3 & 4 & 5 \\
\hline 48. Conoce la historia de la lengua inglesa. & 1 & 2 & 3 & 4 & 5 \\
\hline 49. Habla inglés con mucha fluidez. & 1 & 2 & 3 & 4 & 5 \\
\hline 50. Comprende apropiadamente discursos en inglés. & & & & & \\
\hline
\end{tabular}


http://doi.org/10.15359/ree.25-2.11

http://www.una.ac.cr/educare educare@una.ac.cr

\section{SECCIÓN II: PREGUNTAS ABIERTAS.}

En relación con la misma temática, te agradeceremos que contestes las siguientes preguntas:

1. Considerando el desempeño pedagógico, ¿qué crees que debería caracterizar a un buen profesor o profesora?

2. ¿Cuáles crees son las habilidades lingüísticas que debe dominar un profesor o profesora de inglés?

3. Específicamente, ¿cuáles crees que deberían ser las características de un ‘buen profesor o profesora de inglés?

\section{SECCIÓN III. INFORMACIÓN DE CONTEXTO}

\begin{tabular}{|l|l|}
\hline Nombre \\
\hline Edad & Sexo \\
\hline Año de ingreso a la carrera: & \\
\hline
\end{tabular}

